\title{
Die coriale Flaschenzugnaht
}

\author{
The Corial Pulley Suture
}

Autoren

Institut

\section{H. Kurzen, M. Mazinani, J. Faulhaber, C.-D. Klemke, W. Koenen}

Klinik für Dermatologie, Venerologie und Allergologie, Klinikum Mannheim, Medizinische Fakultät Mannheim der Ruprecht-Karls-Universität Heidelberg

\section{Bibliografie}

Dol $10.1055 / \mathrm{s}-2008-1077671$

Online-Publikation: 26.11.2008

Akt Dermatol 2009; 35:

35-38 @ Georg Thieme Verlag KG Stuttgart · New York ISSN 0340-2541

\section{Korrespondenzadresse}

Prof. Dr. med. Hjalmar Kurzen

Praxis für Dermatologie, Venerologie, Allergologie, Proktologie, Dermatohistologie und medikamentöse Tumortherapie

Kesselschmiedstr. 2 86354 Freising Hjalmar.Kurzen@nexgo.de

\section{Zusammenfassung \\ $\nabla$}

In der Dermatochirurgie erfordert der Verschluss größerer Exzisionsdefekte ein differenziertes Vorgehen. Defekte bis $5 \mathrm{~cm}$ Durchmesser sind je nach Lokalisation in der Regel durch einfache Dehnungsplastiken gut verschließbar, während sich für größere Defekte VY- oder doppelte WYPlastiken bewährt haben. Entscheidend für den Erfolg des Verschlusses ist die spannungsarme und korrekte Adaptation der Wundränder durch eine Coriumsnaht. Während sich bei dünner bis mittelstarker Dermis die horizontale Schmetter-

\section{Einleitung \\ $\nabla$}

Der Verschluss von größeren Exzisionsdefekten, wie z.B. nach histografisch-kontrollierter Entfernung größerer Basaliome, Spinaliome oder nach Exzision von Melanomen mit 1 oder $2 \mathrm{~cm}$ Sicherheitsabstand, erfordert je nach Lokalisation ein differenziertes Vorgehen. Bei Defektgrößen zwischen 2 und $5 \mathrm{~cm}$ ist häufig ein Verschluss mittels einfacher Dehnungsplastik ausreichend. Bei Defekten zwischen 5 und $10 \mathrm{~cm}$ im Durchmesser ist meist ein ästhetisch und funktionell günstiger Verschluss durch eine VY-Plastik oder eine doppelte WY-Plastik möglich. Für noch größere oder unter mäßiggradiger Spannung stehende, schwer verschließbare Defekte ist die Wahl zu treffen zwischen Raffnähten [1], Nahlappen, Rotationsplastiken oder Hauttransplantaten. Für den meist ohne Assistenz operierenden Dermatochirurgen stellt sich dabei die Aufgabe, schnell und kosmetisch befriedigend $\mathrm{zu}$ einem ein- oder zweischichtigen Wundverschluss zu gelangen. Am weitesten verbreitet sind hierbei horizontal oder vertikal gelegene, corial versenkte Einzelknopfnähte, die eine optimale Adaptierung der Wundränder erlauben, allerdings bei größeren Defekten relativ zeitaufwendig und bei Vorliegen von lingsnaht anbietet, hat sich bei dicker und unflexibler Dermis die hier beschriebene vertikale „Flaschenzugnaht“ bewährt. Durch eine doppelte vertikale Schleifenführung mit zentraler Verknotung entsteht ein Flaschenzugeffekt, der zu einer schonenden Annäherung der Wundränder führt, minimales Fremdmaterial in der Wunde belässt und durch Platzierung des Knotens in der Tiefe der Wunde ein optimales kosmetisches Ergebnis produziert. Die Flaschenzugnaht vereint die Vorteile einer fortlaufenden Coriumsnaht mit der höheren Sicherheit der Einzelknopfnähte.

Spannung an den Wundrändern z.T. schwierig zu knüpfen sind. Eine sehr nützliche Modifikation der Coriumsnaht ist die sog. Schmetterlingsnaht nach Breuninger [2], mit der insbesondere bei dünnem und mittelstarkem Corium ein sehr schöner und schneller Wundverschluss, häufig ohne Notwendigkeit einer Oberflächennaht, gelingt. Ist jedoch das Corium relativ dick, wie z.B. am Rücken oder an der Schulter, bereitet die korrekte Ausführung der Schmetterlingsnaht im $45^{\circ}$-Winkel zum Wundrand aufgrund der fehlenden Flexibiltät des Coriums und der damit erschwerten Nadelführung häufig Probleme. In dieser Situation hat sich die hier beschriebene coriale „Flaschenzugnaht“ sehr bewährt [3].

\section{Technik}

Die Flaschenzugnaht ist eine vertikale gestellte, doppelläufige, versenkte Coriumsnaht in Form einer 8 mit zentral gelegenem Knoten. Die Nadelführung beginnt an den äußeren Rändern des Defektes, ca. $0,5 \mathrm{~cm}$ von der Spindelspitze wie bei einer klassischen vertikalen Coriumnaht von subkutan durch das Corium. Die Fadenführung hat ihren höchsten Punkt ca. $0,5 \mathrm{~cm}$ von der 
Wundlippe entfernt, tritt im oberen Corium in die Wunde aus und tritt auf gleicher Höhe wieder auf der gegenüberliegenden Wundseite in das obere Corium ein. Dort verläuft sie ansteigend bis zum höchsten Punkt, wieder 0,5 cm von der Wundlippe entfernt nach unten und tritt im subkutanen Fettgewebe in die Wunde aus. Bei einer Einzelknopfnaht würde nun geknotet werden ( Abb. 1, Abb. 2 b), bei der Flaschenzugnaht wird die Nadel mit Faden nun aber 0,5-1 cm weiter zum Zentrum des Defekts verschoben und parallel zur ersten Schleife geführt. $\mathrm{Zu}$ achten ist hierbei darauf, dass die Nadel beim letzten Austritt in die Wunde zwischen den beiden Schleifen herauskommt ( $\bullet$ Abb. 1, Abb. 2c-d). So kann der Faden mit dem freien Ende, das ebenfalls zwischen den beiden Schleifen zu liegen kommen soll, verknotet werden ( $\bullet$ Abb. 2 e). Durch das vorsichtige Zusammenziehen der Fadenenden werden die Wundränder problemlos adaptiert ( $\bullet$ Abb. 2f). Der hierbei durch das mehrfache Umleiten des Fadens stattfindende Flaschenzugeffekt erlaubt die atraumatische Annäherung der Wundränder, auch wenn diese unter Spannung stehen [4]. Das Ausmaß der Spannung sollte hierbei natürlich so gering wie möglich gehalten werden, wofür wie bei allen Verschlusstechniken sowohl eine geeignete Schnittführung als auch eine ausreichende Mobilisation der Wundränder notwendig ist. Sind beide Spindelspitzen mit Flaschenzugnähten versorgt, bleibt je nach Defektgröße ein Restdefekt, der nach Maßgabe der Größe entweder mit einzelnen vertikal versenkten Coriumnähten versorgt wird oder mit weiteren Flaschenzugnähten sukzessive verschlossen wird.

\section{Diskussion}

Wie von Hohenleutner und Mitarbeitern gezeigt werden konnte, ist das kosmetische Ergebnis bei alleinigem corialem Verschluss dem unter Verwendung einer Oberflächennaht (z.B. Einzelknopfnaht) zumindest nicht unterlegen. Es hat jedoch die Vorteile, dass es schneller durchführbar ist und dass keine Fäden gezogen werden müssen. Wunddehiszenzen oder hypertrophe Narben kamen bei alleinigem corialem Verschluss nicht häufiger vor [5]. Im Vergleich zur einzeln geknüpften Coriumsnaht, hat die Flaschenzugnaht den Vorteil, dass weniger Fremdmaterial und Knoten in der Haut verbleiben. Die Nahttechnik verteilt den Gewebezug besser und vermeidet dadurch Nekrosen oder ein Reißen des Nahtmaterials. Bei optimaler Durchführung ist bei kleineren Defekten keine Oberflächennaht notwendig, eine Versorgung mit Klebestreifen (z.B. Steristrips) ist ausreichend ( Abb. 2f). Sollten nach Abschluss der Flaschenzugnaht doch kleine Adaptationslücken übrig geblieben sein, was insbesondere bei größeren Defekten leicht der Fall sein kann, wird eine dünne oberflächliche Naht entweder in Einzelknopftechnik oder auch fortlaufend zur Korrektur verwendet. Durch Legen des höchsten Schleifenpunktes 0,5 cm von der Wundlippe entfernt, entsteht beim Zusammenziehen der gewünschte Evertierungseffekt, d.h. die Wundlippen sind nach außen konvex bzw. bilden einen „Hügel“. Dadurch kommt die endgültige Narbe (nach 6-12 Monaten) nach Ende der normalen Schrumpfung exakt ins Hautniveau. Dieser Effekt kann wie bei der Schmetterlingsnaht durch eine spezielle, den Wundrand nach lateral unterminierende Skalpellführung (45-60'-Winkel) optimiert werden.

Ein Vorteil im Vergleich zur Schmetterlingsnaht ist das fehlende Zusammenziehen oder „Verschrumpeln“ der Wundränder, das durch die horizontale Anlage der Schmetterlingsnaht häufig entstehen kann. Die Schmetterlingsnaht ist insbesondere bei sehr

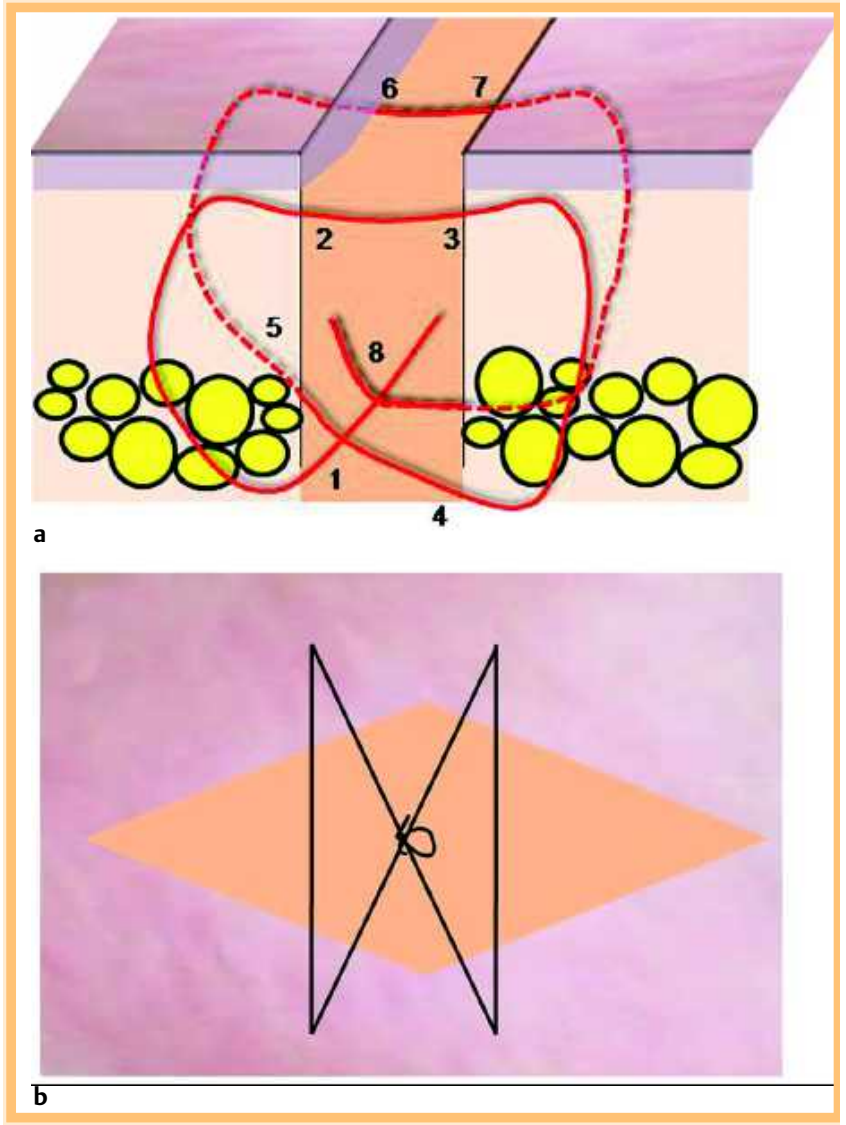

Abb. 1 Schematische Darstellung des Fadenverlaufs bei der corialen Flaschenzugnaht. a Die Nadelführung beginnt von subkutan (1) durch das Corium, tritt im oberen Corium in die Wunde aus (2), tritt auf gleicher Höhe wieder auf der gegenüberliegenden Wundseite in das obere Corium ein (3), nach unten und tritt im subkutanen Fettgewebe wieder in die Wunde aus (4). Die Nadel mit Faden wird 0,5-1 cm weiter zum Zentrum des Defekts verschoben und parallel zur ersten Schleife geführt (5-8). Die Nadel kommt beim letzten Austritt in die Wunde zwischen den beiden Schleifen heraus. b Blick von oben auf eine durch 4 Flaschenzugnähte verschließbare spindelförmige Wunde. Der Knoten der Flaschenzugnaht liegt zentral zwischen den beiden Schleifen des Fadens.

dickem Corium, wie es beispielsweise am Rücken häufig der Fall ist, aufgrund der mangelnden Flexibilität weniger gut geeignet. Der komplexe, eher horizontale Fadenverlauf der Schmetterlingsnaht ist deutlich schwieriger zu bewerkstelligen als ein vertikaler wie bei der Flaschenzugnaht. Besonders geeignet ist die „Flaschenzugnaht“ zur sicheren und schnellen Verankerung der Y-Dreieckspitze bei Ausführung einer VY- oder doppelten WY-Plastik ( Abb.3). Diese wird bei Ausführung der ersten Schleife nach dem 1. Austritt der Nadel aus dem Wundrand auf Höhe des oberen Corium im mittleren Bereich horizontal durchstochen. Der weitere Verlauf der Naht ist wie oben beschrieben. Die 2. Schleife kommt vor die Dreiecksspitze zu liegen, der Knoten wird an der 1. Eintrittsstelle des Fadens im Subkutanbereich in Richtung des Y-Schenkels zusammengezogen.

Als Nahtmaterial für die Flaschenzugnaht eignen sich unterschiedliche resorbierbare Materialien, in unseren Händen haben sich $0-0$ bis 3-0 geflochtene Fäden (z.B. Serafit ${ }^{\circledR}$ oder Vicryl ${ }^{\circledR}$ ) bewährt. Für den Oberflächenverschluss reichen für Einzelknopfnähte sehr viel dünnere Fäden, in der Regel 4-0 bis 5-0 Monofil (z. B. Seralon ${ }^{\circledR}$ ). Alternativ kann die Adaptation per Intrakutannaht perfektioniert und gesichert werden. 

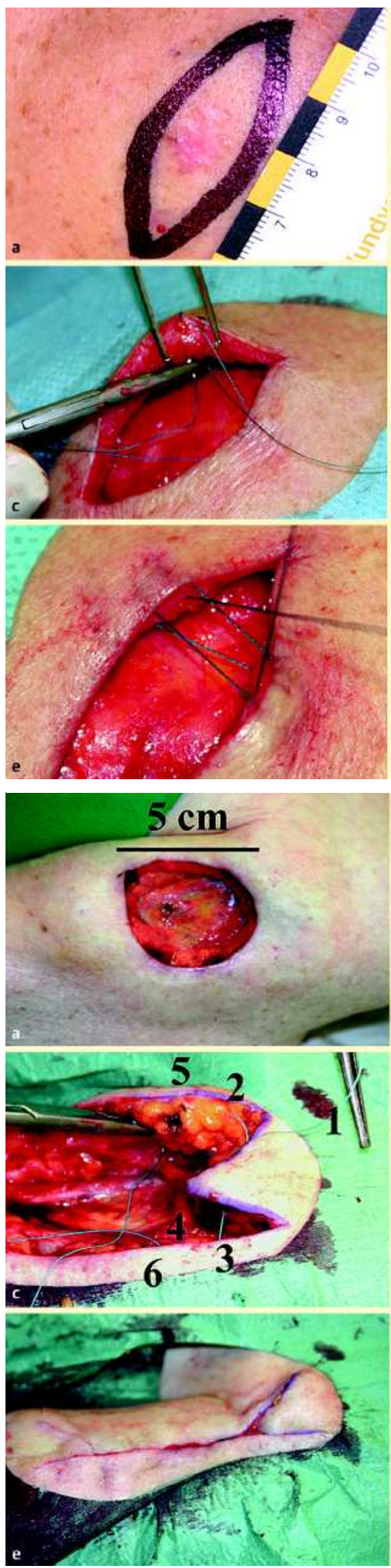
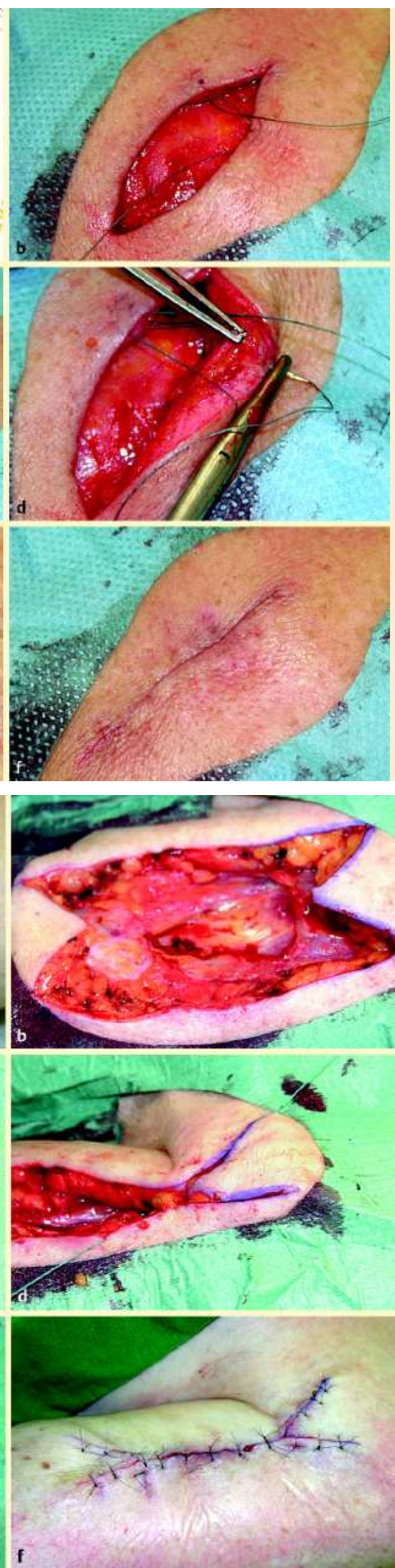

Abb. 2 Dehnungsplastik zum Verschluss eines einfachen Exzisionsdefektes mithilfe der corialen Flaschenzugnaht. a Superfizielles Basaliom am rechten Schulterblatt, eingezeichnete spindelförmige Schnittführung zur Exzision mit 2 mm Sicherheitsabstand. b Exzisionsdefekt. Die erste horizontale Schleife ist bereits wie für eine vertikale coriale Einzelknopfnaht erfolgt (Faden: Terylene ${ }^{\circledR} 2-0$, zur Demonstration, für endgültigen Verschluss wurde 2-0 Serafit ${ }^{\circledR}$ verwendet). c Die zweite Schleife erfolgt parallel zur ersten: Einstich von unten. d Zweiter Teil der 2. Schleife: Einstich von oben, Ausstich zentral unten (Pinzette). e Fadenführung in Form einer Acht. Der Knoten kommt zentral zu liegen. $f$ Die Wunde wurde durch 3 coriale Flaschenzugnähte vollständig verschlossen. Eine Hautnaht ist nicht notwendig.

Abb. 3 Doppelte WY-Plastik zum Verschluss eines großen Exzisionsdefektes mit Hilfe der corialen Flaschenzugnaht. a $5 \mathrm{~cm}$ durchmessender Exzisionsdefekt an der linken Schulter bei Z.n. Lentigo maligna. b Defekt nach Zuschnitt für Verschluss durch doppelte WY-Plastik (Länge $10 \mathrm{~cm}$ ). c Fadenführung für coriale Flaschenzugnaht zur Demonstration mit 2-0 Terylene ${ }^{\circledR}$. Nach Anfertigung der Fotos wurde der Terylene ${ }^{\circledR}$-Faden durch 2-0 Serafit ${ }^{\circledR}$ ersetzt. Freies Fadenende (1), erste Schleife mit Fadenaustritt aus dem Corium (2), Faden quer durch die Spindelspitze und Eintritt in das Corium (3), Austritt des Fadens in der Tiefe der Wunde (4), zweite Schleife des Flaschenzugs mit Austritt der Nadel aus dem Corium (5). Die zweite Schleife wird vollendet auf der gegenüberliegenden Seite mit Eintritt in das Corium (6) und Austritt aus der Tiefe der Wunde. Der Knoten wird zwischen den beiden Schleifen des Flaschenzugs zentral geknüpft. d Problemlose Adaption der Wundränder. e Vollständig adaptierte Wundränder. $\mathbf{f}$ Abschluss der Operation mit 5-0 Seralon Einzelknopfnähten. 


\section{Abstract}

\section{The Corial Pulley Suture \\ $\nabla$}

The closure of larger incisional defects in dermatosurgery requires different techniques according to the localization of the defect and the texture of the skin. Depending on the localization, defects up to $5 \mathrm{~cm}$ diameter can easily be closed by extension plastic while larger defects often require VY- or double WY-plastics. A crucial point for the esthetical outcome of the procedure is the choice of the corial suture technique. Defects in localizations with thin to moderately thick corium can be closed with the horizontal butterfly suture. However, in localizations with thicker corium, the corial pulley suture is more advantageous. Two vertical suture loops, knotted centrally produce a block and tackle effect which allows atraumatic approximation of the wound margins, leaves minimal foreign material in the wound and places the knot in depth of the wound thus avoiding granuloma formation. Altogether the cosmetical results of this simple technique are excellent. The advantages of a corial running suture are combined with the increased security of a multiple knot approach.

\section{Literatur}

1 Koenen W, Kurzen H, Goerdt S, Faulhaber J. Defect reduction by serial tightening of loop sutures. J Dtsch Dermatol Ges 2007; 5: 330-331

2 Breuninger $H$. Double butterfly suture for high tension: a broadly anchored, horizontal, buried interrupted suture. Dermatol Surg 2000; 26: $215-218$

3 Whalen JD, Dufresne RG, Collins SC. Surgical pearl: the modified buried dermal suture. J Am Acad Dermatol 1999; 40: 103-104

4 Austin BR, Henderson RA. Buried tension sutures: force-tension comparisons of pulley, double butterfly, mattress, and simple interrupted suture patterns. Vet Surg 2006; 35: $43-48$

5 Hohenleutner U, Egner $N$, Hohenleutner S, Landthaler M. Intradermal buried vertical mattress suture as sole skin closure: evaluation of 149 cases. Acta Derm Venereol 2000; 80: 344-347 